\title{
Trends in head and neck radiology
}

\author{
Sabrina Kösling \\ Received: 22 October 2010 /Revised: 30 November 2010 /Accepted: 2 December 2010 /Published online: 14 January 2011 \\ (C) European Society of Radiology 2011
}

\begin{abstract}
Trends in clinical research of head and neck radiology are well described within four articles published in the European Radiology in the last 2 years. One constant top issue is to gain more, better or new morphological information by so-called "conventional" methods (CT, MRI) with thinsliced imaging including special reconstructions. The addition of functional or metabolic methods (e.g. diffusion-weighted MRI, dynamic contrast-enhanced MRI, positron emission tomography) to anatomical imaging is a further general trend in oncological radiology over the last few years and has been invaluable in head and neck radiology.
\end{abstract}

Keywords Head and neck radiology Clinical research . Trends $\cdot$ Anatomical imaging $\cdot$ Functional methods

\section{Introduction}

The head and neck region is an area of difficult and often subtle anatomy. Therefore, one constant top issue of clinical research in head and neck radiology is to gain more, better or new morphological information by so-called "conventional" methods (CT, MRI) with thin slices, special reconstructions or sequences. The results of these studies influence the daily radiological work directly. It is known that optimal head and neck findings require investigations with high spatial resolution and profound anatomical understanding.

Even today, rare variants or helpful signs are newly published. For example, Hermans and Rensburg [1] identified aberrant petrous bone channels on $\mathrm{CT}$, which never have been described before. By MRI they could show a vessel running within this channel. From analysing the embryological development of the dural sinuses they concluded that this vessel corresponds most probably to a persistent embryonic vein. Such knowledge of anatomical variants is important to avoid a misinterpretation as pathological structures.

\section{S. Kösling $(\bowtie)$}

Department of Diagnostic Radiology, University of Halle,

E-Grube-Str. 40,

06097 Halle, Germany

e-mail: sabrina.koesling@medizin.uni-halle.de
In blunt or penetrating orbital injuries it is sometimes clinically and radiologically difficult to decide whether or not there has been an open globe injury. Based on thin sliced CT, Kim et al. [2] recommend the measurement of anterior chamber depth (posterior margin of the cornea to anterior margin of the lens should be measured in the midline of the tangent through the anterior lens). The authors found that a difference of the anterior chamber depth between the injured and non injured eye of more than $0.4 \mathrm{~mm}$ is a very helpful diagnostic criterion which carries excellent specificity.

The addition of functional or physiological and metabolic methods (e.g. diffusion-weighted MRI (DWI), dynamic contrastenhanced MRI, positron emission tomography (PET)) to anatomical imaging is a general trend in radiology in the last years and one of crucial importance in head and neck radiology. These methods are mainly applied to improve the characterisation of lesions and lymph nodes, especially in patients with an oncological background. Although the preliminary results are quite encouraging, the influence of these studies in routine clinical work is only slowly emerging. One major problem is that standardization is often missing. Single groups work with different gradient strengths for DWI, obtain different thresholds of apparent diffusion coefficient (ADC) or take different protocols for dynamic contrast-enhanced MRI (flow, delay, time and interval of images acquisition); such variations at a local level mean that results from different centres are not strictly comparable. Beside limited spatial resolution, another point is that functional methods still cannot deliver the exact histological information (with determination of the one of the sometimes numerous subtypes) which is essentially needed for treatment planning of malignancies. Hence biopsy is still often required. Before any therapy is given for malignant neoplasms, the main value of functional methods should be seen in obtaining a baseline for post-therapeutic follow-up and in supporting the decision for observation in selected cases of possibly benign lesions. The value of functional methods should be especially seen in posttherapeutic follow-up. For instance, such investigations can help predict likely responders from non-responders. From all the studies about functional imaging published in European Radiology in the last 2 years, two will be discussed further.

King et al. [3] and Vandecaveye et al. [4] stressed the necessity of an early identification of non-responders to radiotherapy or chemoradiotherapy for modification or change of treatment 
regimens in patients with head and neck squamous cell carcinoma. In this early post-treatment phase previous radiological assessment has been described as suboptimal: Treatmentinduced inflammation may hamper the accuracy of PET-CT. Volumetric analysis with anatomical imaging (CT, MRI) has shown variable results. The differentiation between residual cancer and benign post-treatment mass is difficult. Moreover, postradiation mucositis or crust may hinder endoscopic examination.

DWI is a quickly performed and widely available MRI technique which visualises the Brownian movement of water; it can often be included within a series of MR sequences with little additional time penalty. In tumours it is hypothesized that a higher cell density (malignant tissue) leads to a stronger restraint of diffusion corresponding with a low $\mathrm{ADC}$ value. Among others this principle is used for the prediction and monitoring of treatment response in malignancies undergoing a non-surgical therapy. King et al. [3] and Vandecaveye et al. [4] analysed whether a change in $\mathrm{ADC}$ could be an early diagnostic biomarker to solve the problematic evaluation of residual tissue in the tumour and lymph node bed. Vandecaveye et al. [4] examined 30 patients before, and at the $2 \mathrm{nd}$ and 4 th week during radiochemotherapy by anatomical MRI and DWI and studied changes in tumour volume and ADC. Recurrence and complete response was measured against a 2 year follow-up. The ADC change of the primary tumour and nodal metastases was significantly lower in patients in whom a recurrence developed after therapy than in those patients with locoregional control. While the change in ADC correlated significantly with the 2 year local control, the change in volume did not. Thus DWI allowed a more accurate prediction of response than anatomical imaging alone. King et al. [3] investigated 50 patients and correlated $\mathrm{ADC}$ values (obtained before treatment, 2 weeks after starting of treatment and 6 weeks after the end of treatment) with locoregional failure, which occurred in 20 patients (Fig. 1). A significant correlation was only found between locoregional failure and a decrease of $\mathrm{ADC}$ value on post-treatment images. Although the DWI technique employed was slightly different in these two studies, a serial decrease of ADC values from pretreatment until early post-treatment time seems to be a good and readily obtainable marker for non response to no-surgical therapy in squamous cell carcinoma of the head and neck. It is to be hoped, that in the future, a standardisation of different functional methods will lead to a better comparison of studies and an easier handling in practical work.
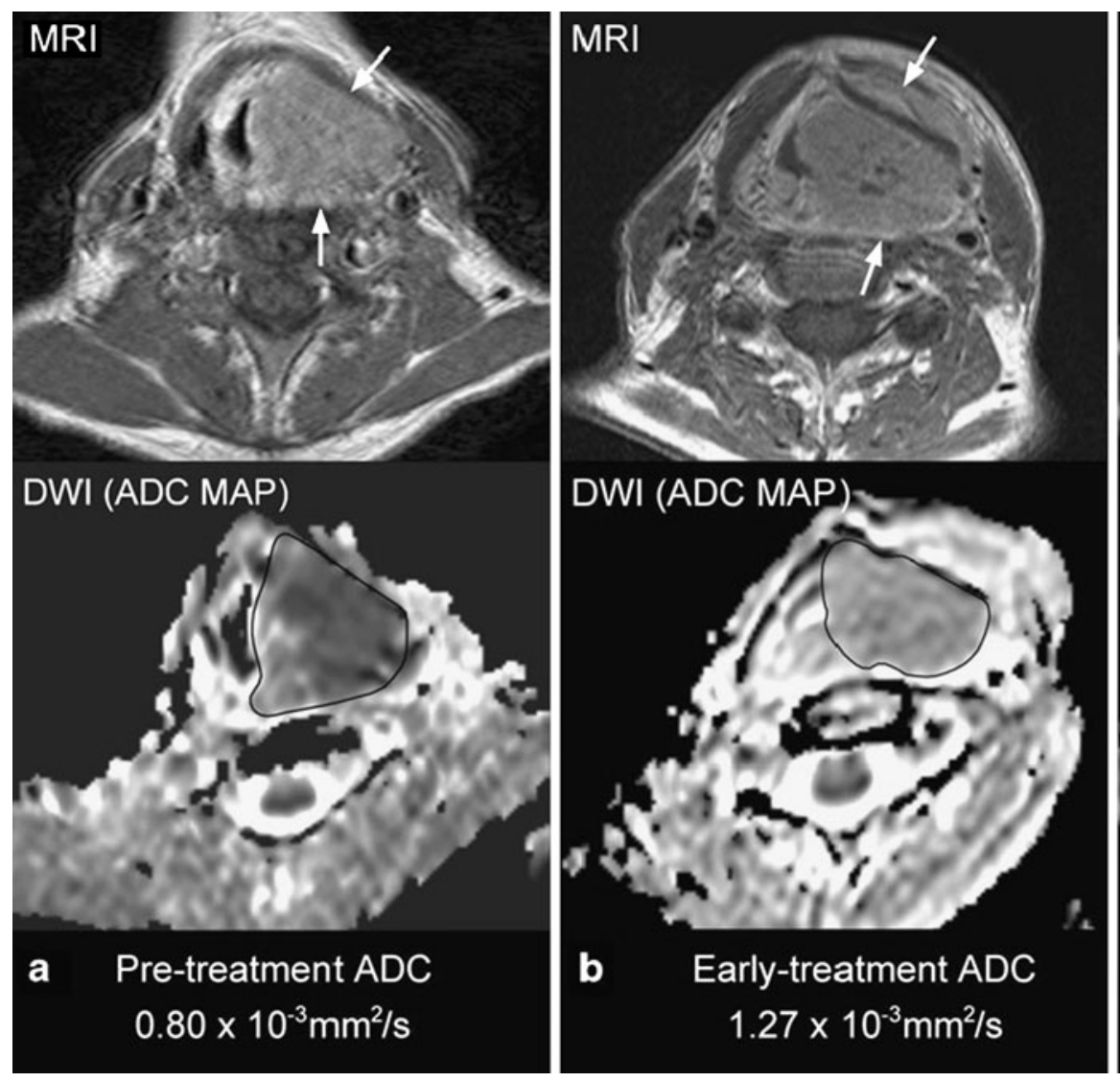
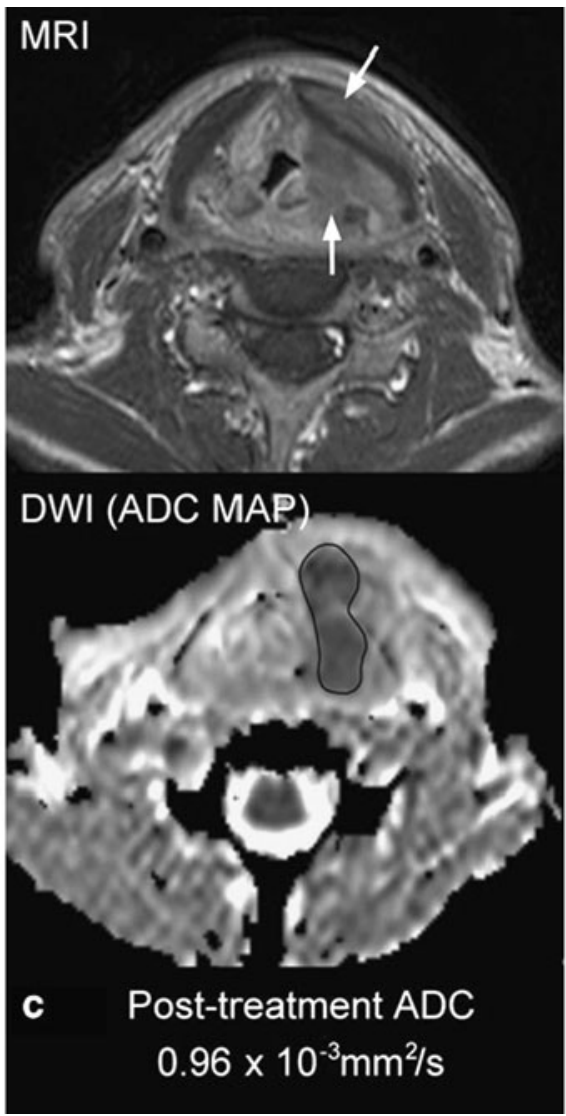

Fig. 1 Axial T1-weighted post-contrast MRI (upper row) and ADC maps (lower row) of a hypopharyngeal tumour (arrows) showing a rise of ADC from pre-treatment images (first column) to early-treatment images (middle column) followed by a fall on post-treatment images (last column). This pattern is highly suggestive for local failure. It was proven by histology in this case. (with permission from King AD, Mo FKF, Yeung DKW et al. (2010) Sqaumous cell carcinoma of the head and neck: diffusion-weighted MR imaging for prediction and monitoring of treatment response. Eur Radiol 20: 2213-2220) 


\section{References}

1. Hermans R, van Rensburg LJ (2009) An aberrant vascular channel in the petrous bone: persistent lateral capital vein? Eur Radiol 19:2958-2964

2. Kim SY, Lee JH, Lee YJ et al (2010) Diagnostic value of the anterior chamber depth of a globe on CT for detecting open-globe injury. Eur Radiol 20:1079-1084
3. King AD, Mo FKF, Yeung DKW et al (2010) Sqaumous cell carcinoma of the head and neck: diffusion-weighted MR imaging for prediction and monitoring of treatment response. Eur Radiol 20:22132220

4. Vandecaveye V, Dirix P, De Keyzer F et al (2010) Predictive value of diffusion- weighted magnetic resonance imaging during chemoradiotherapy of head and neck squamous cell carcinoma. Eur Radiol $20: 1703-1714$ 